\title{
Pituitary Macroadenoma and Visual Impairment: Postoperative Outcome Prediction with Contrast-Enhanced FIESTA
}

\author{
(DS. Hisanaga, (DS. Kakeda, (D). Yamamoto, DK. Watanabe, (D). Moriya, (D). Nagata, (D). Fujino, (D). Kondo, (DS. Nishizawa, and \\ (i) Y. Korogi
}

\begin{abstract}
BACKGROUND AND PURPOSE: Contrast-enhanced FIESTA can depict anterior optic pathways in patients with large suprasellar tumors. We assessed whether the degree of kink in the optic nerve at the optic canal orifice on contrast-enhanced FIESTA correlates with the postoperative improvement of visual impairment in patients with pituitary macroadenoma.
\end{abstract}

MATERIALS AND METHODS: Thirty-one patients with pituitary macroadenoma who underwent preoperative MR imaging and an operation were evaluated. We measured the optic nerve kinking angle on sagittal oblique contrast-enhanced FIESTA parallel to the optic nerve; the optic nerve kinking angle was defined as the angle between a line parallel to the planum sphenoidale and a line parallel to the intracranial optic nerve at the optic canal orifice. We used logistic regression analyses to determine whether the clinical (sex, age, and duration of symptoms) and imaging (tumor height, chiasmal compression severity, hyperintense optic nerve on $\mathrm{T} 2 \mathrm{Wl}$, and optic nerve kinking angle) characteristics were associated with the postoperative improvement (good-versus-little improvement) of visual acuity disturbance and visual field defect.

RESULTS: There were 53 impaired sides before the operation: 2 sides with visual acuity disturbance alone, 25 with visual field defect alone, and 26 with both. After the operation, good improvement was found in 17 of the 28 sides with visual acuity disturbance and in 32 of the 51 sides with visual field defects. Only the optic nerve kinking angle was significantly associated with good improvement of the visual acuity disturbance $(P=.011)$ and visual field defect $(P=.002)$.

CONCLUSIONS: The degree of the optic nerve kinking angle was an independent predictor of postoperative improvement, indicating that irreversible damage to the optic nerve may be associated with its kinking at the optic canal orifice.

ABBREVIATIONS: $\mathrm{CE}=$ contrast-enhanced; $\mathrm{ON}=$ optic nerve; $\mathrm{ONKA}=$ optic nerve kinking angle; $\mathrm{VAD}=$ visual acuity disturbance; $\mathrm{VFD}=$ visual field defect

im patients with pituitary macroadenoma, the presence of visual impairment, namely visual acuity disturbance (VAD) and visual field defect (VFD), is a major indication for surgical treatment. ${ }^{1} \mathrm{Al}-$ though several studies have reported that the VFD in some $10 \%-$ $20 \%$ of cases was not improved with an operation, ${ }^{2-4}$ the predictors for the postoperative improvement of visual impairment are controversial. Furthermore, few studies have evaluated the ability of MR imaging to predict postoperative improvement in visual impairment. Watanabe et $\mathrm{al}^{5}$ reported that a hyperintense optic nerve $(\mathrm{ON})$ on MR imaging predicted persistence of visual impairment after the

Received January 11, 2017; accepted after revision June 6.

From the Departments of Radiology (S.H., S.K., K.W., J.M., Y.K.), Neurosurgery (J.Y., S.N.), Ophthalmology (T.N., H.K.), and Preventive Medicine and Community (Y.F.), University of Occupational and Environmental Health School of Medicine, Kitakyushu, Japan.

Please address correspondence to Shingo Kakeda, MD, Department of Radiology, University of Occupational and Environmental Health, 1-1 Iseigaoka,

Yahatanishi-ku, Kitakyushu 807-8555, Japan; e-mail: kakeda@med.uoeh-u.ac.jp

三 Indicates article with supplemental on-line tables.

http://dx.doi.org/10.3174/ajnr.A5394 operation, but the group of patients in whom VAD and/or VFD persisted after an operation was quite small (only 4 patients). ${ }^{5}$

Bitemporal hemianopsia is a well-known VFD associated with pituitary macroadenomas that compress the chiasm. However, a recent report revealed that complete bitemporal hemianopsia associated with pituitary macroadenoma was rare. ${ }^{6}$ The authors also demonstrated that $44.9 \%$ of patients with VFDs had nontemporal defects, suggesting that it is not uncommon to have extrachiasmal optic pathway involvement rather than chiasmal compression alone. Furthermore, in large suprasellar tumors, such as tuberculum sellae meningiomas, the ONs are sometimes compressed at the optic canal orifice, and some investigators suggest that $\mathrm{ON}$ compression at the optic canal orifice may be the main cause of visual impairment and asymmetric visual loss. ${ }^{7,8}$

Balanced steady-state free-precession techniques, such as fast imaging employing steady-state acquisition, constructive interference in steady state, fast imaging with steady-state free precession, and balanced fast-field echo, can provide strong $\mathrm{T} 2$ contrast, which emphasizes CSF signals. ${ }^{9-12}$ FIESTA also shows contrast enhancement due to $\mathrm{T} 1$ shortening caused by administration of 
gadolinium-based contrast material, because signal intensity on FIESTA is partially T1-dependent. ${ }^{13,14}$ Therefore, in patients with skull base tumors, gadolinium-based contrast material can increase the relative contrast between the enhanced tumor and the cranial nerves using FIESTA. A previous study reported that contrast-enhanced FIESTA (CE-FIESTA) was a useful MR imaging method for performing preoperative evaluation of suprasellar tumors, because CE-FIESTA can depict segments of the optic pathways in patients with large suprasellar tumors. ${ }^{5}$

When using CE-FIESTA, we frequently observe kinking in the $\mathrm{ON}$ at the optic canal orifice because of compression by pituitary macroadenomas, prompting the hypothesis that this feature on CE-FIESTA might be useful for determining the postoperative prognosis of visual impairment. Thus, the aim of the current study was to determine whether the degree of ON kink at the optic canal orifice correlates with the postoperative improvement of visual impairment in patients with pituitary macroadenoma.

\section{MATERIALS AND METHODS \\ Patient Selection}

The institutional review board approved this retrospective study and waived informed consent. By searching and reviewing the operative records in our institution between May 2007 and May 2015, we selected 34 consecutive patients with pituitary adenomas who underwent an operation and received a pathologically proved diagnosis. A single radiologist (S.H., with 4 years' experience in radiology) included patients in this study provided they met all of the following: 1) They had a pituitary macroadenoma that tightly touched or compressed the optic pathways on MR imaging; 2) they had undergone preoperative brain MR imaging examinations with our standard brain MR imaging protocol, which included CE-FIESTA; 3) after an operation, the tumor reduction and compression of the optic chiasm resolved; and 4) formal ophthalmology consultations before and after the surgical treatment were available. Three of the 34 patients were excluded from analysis for the following reasons: One was diagnosed with a pituitary apoplexy, and one had a history of an operation due to a brain tumor. One patient with optic disc pallor and atrophy on ophthalmologic examinations before the operation was also excluded because it predicted poor outcome. ${ }^{15}$ In this patient, the $\mathrm{VAD}$ and/or VFD persisted after the operation. Thirty-one patients were ultimately included in the final analysis ( 16 women, 15 men; mean age, $54.6 \pm 16.9$ years; age range, $18-87$ years). The surgical approach was transsphenoidal in all patients.

Before the operation, 29 (94\%) of 31 patients had VAD and/or VFD. The ophthalmologic examinations showed VADs in 18 patients (bilateral in 10 patients and unilateral in 8). Regarding VFDs, the ophthalmologic examinations showed bilateral temporal hemianopia in 17 patients, unilateral temporal hemianopia in 5 patients, bilateral superior temporal defects in 3 patients, bilateral homonymous hemianopia in 2 patients, and bilateral central scotoma in 1 patient. There were 53 impaired sides in 29 patients: 2 sides (4\%) with VAD alone, 25 (47\%) with VFD alone, and 26 (49\%) both with VAD and VFD. Two patients who had neither VAD nor VFD underwent surgical treatment for chiasmal compression due to pituitary macroadenoma; 1 patient also showed tumor progression during follow-up MR imaging.

\section{MR Imaging Data Acquisition}

All MR imaging studies were performed with a Signa Excite 3T scanner (GE Healthcare, Milwaukee, Wisconsin) using a dedicated 8-channel phased array coil (USA Instruments, Aurora, Ohio). Gadodiamide (Omniscan; GE Healthcare, Piscataway, New Jersey) or gadopentetate dimeglumine (Magnevist; Bayer HealthCare Pharmaceuticals, Wayne, New Jersey) was administered at $0.1 \mathrm{mmol} / \mathrm{kg}$ of body weight as the intravenous contrast agent. We used the following parameters for the FIESTA sequence: TR/TE, 5.4/2.4 ms; acquisition, 2 times; flip angle, 50 bandwidth, $\pm 62.5 \mathrm{kHz}$; matrix, $224 \times 224 ; 100 \%$ image; section thickness, $0.8 \mathrm{~mm}$; FOV, $14 \times 14 \mathrm{~cm}$; resolution, $0.6 \times 0.6 \times 0.8$ $\mathrm{mm}$; and imaging time, 5 minutes 6 seconds. All CE-FIESTA data were acquired in the coronal plane. In addition, all patients underwent our standard brain MR imaging protocol for suprasellar tumors, including coronal T2WI. We used the following imaging parameters for the coronal T2WI: TR/TE, 4000/85 ms; flip angle, $90^{\circ}$; bandwidth, $62.5 \mathrm{kHz}$; section thickness, $3 \mathrm{~mm}$; matrix, $512 \times$ 224; FOV, $18 \times 18 \mathrm{~cm}$; imaging time, 2 minutes 16 seconds.

\section{Ophthalmologic Examination and Evaluation}

Ophthalmologic examinations were performed by ophthalmologists and consisted of testing the patient's visual acuity with the best-corrected visual acuity for both eyes, funduscopy, and measuring intraocular pressure for the diagnosis of concomitant disorders, such as glaucoma or/and cataracts. VAD was positive when the visual acuity with the best-corrected visual acuity was $<20 / 50$ vision on the Snellen Eye Chart. ${ }^{16}$ The visual fields were determined with the Goldmann perimeter (isopters: V/4, III/4, $\mathrm{I} / 4, \mathrm{I} / 3, \mathrm{I} / 2$, and $\mathrm{I} / \mathrm{l}){ }^{17}$

The pre- and postoperative VAD and/or VFD were compared, and the patients were divided into 2 subgroups based on the improvement between the 2 points: good improvement versus little improvement. For patients who had undergone an ophthalmologic examination after the operation, the results of the most recent examination were included in this analysis. For VADs, good postoperative improvement was defined when the postoperative visual acuity with the best-corrected visual acuity was 20/50 vision or better on the Snellen Eye Chart, whereas little improvement was defined when the postoperative visual acuity with the bestcorrected visual acuity was $<20 / 50$ vision, despite the absence of any concomitant disorders. For VFDs, good postoperative improvement was defined as either the disappearance of the VFD or the persistence of the VFD with I/3, I/2, or I/1, whereas little improvement was defined as the persistence of the VFD with V/4, III/4, or I/4.

\section{Image Interpretation}

Two radiologists (radiologists A [J.M.] and B [K.W.], with 16 and 8 years' experience in neuroradiology, respectively), who were unaware of the clinical findings, independently evaluated the CEFIESTA and coronal T2WI. To assess the CE-FIESTA findings, we used 3 cross-sectional images (coronal, axial, and sagittal) reconstructed with a section thickness of $1 \mathrm{~mm}$ without a gap. First, the 2 radiologists evaluated the CE-FIESTA data for depiction of the anterior optic pathways, and this was scored as either visible or invisible. Simultaneously, the radiologists also blindly reviewed 


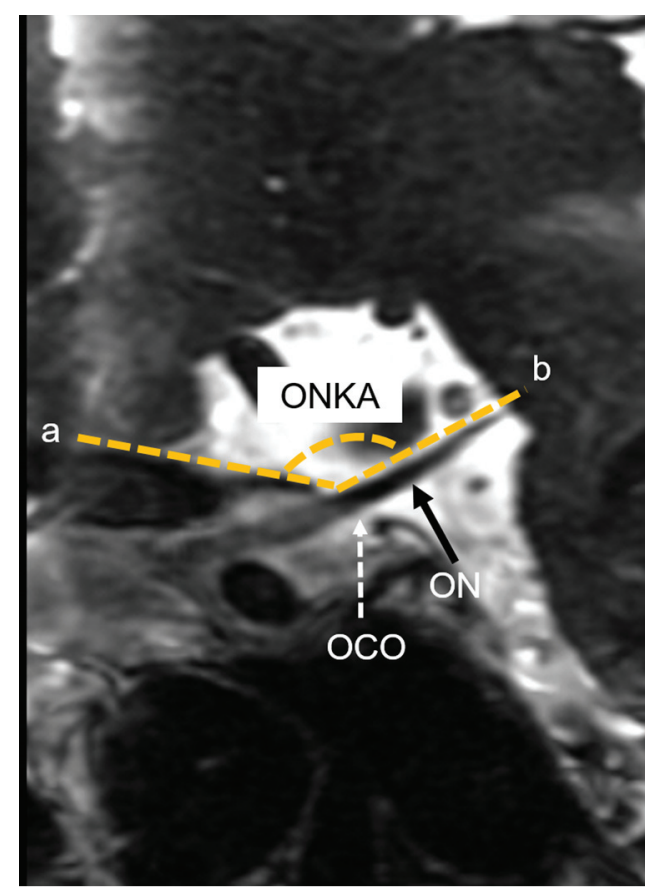

FIG 1. A sagittal oblique CE-FIESTA from a subject with normal brain MR imaging findings shows the optic nerve kinking angle. $a=$ line extending across the anterior cranial fossa, $b=$ line through the intracranial optic nerve. OCO indicates optic canal orifice.

abnormal signal intensity in the anterior optic pathways (bilateral ONs, optic chiasm, and bilateral optic tracts) on CE-FIESTA images. The signal intensity of the optic chiasm and tracts could not be sufficiently evaluated with T2WI because it could not depict the optic chiasm and tracts when they were markedly thinned and/or deviated due to tumor compression. Thus, for the T2WI, the radiologists reviewed the signal intensity only in the bilateral ONs. The radiologists also classified the degree of chiasmal compression on CE-FIESTA into 2 grades (mild or moderate compression, $<5 \mathrm{~mm}$ of optic chiasm displacement; and severe compression, $\geq 5 \mathrm{~mm}$ of optic chiasm displacement). ${ }^{6}$ The cephalocaudal diameter of each tumor was also measured on sagittal CE-FIESTA images. According to the information above, for the analyses, we used the final judgments by 2 radiologists in consensus and the mean measurements by the radiologists.

\section{Measurement of the Optic Nerve Kinking Angle at the Optic Canal Orifice}

Two radiologists (J.M. and K.W.) measured the optic nerve kinking angle (ONKA) on sagittal oblique mages parallel to the $\mathrm{ON}$ reconstructed from CE-FIESTA image data. The ONKA was defined as the angle between a line parallel to the planum sphenoidale and a line parallel to the intracranial $\mathrm{ON}$ at the optic canal (Fig 1). Although we found susceptibility artifacts on CE-FIESTA related to the skull base, no cases showed any degradation of the image quality for measuring the ONKA.

\section{Statistical Analyses}

All the statistical analyses were performed with the SPSS program (IBM, Armonk, New York). $\kappa$ statistics were used to evaluate the level of interobserver agreement of scores from the 2 radiologists. The strength of agreement was considered fair for $\kappa$ values of
Table 1: The evaluation of chiasmal compression by 2 radiologists

\begin{tabular}{lccc}
\hline & \multicolumn{3}{c}{ Radiologist A } \\
\cline { 2 - 4 } Radiologist B & Mild or Moderate & Severe & Total \\
\hline Mild or moderate & 7 & 2 & 9 \\
Severe & 4 & 18 & 22 \\
Total & 11 & 20 & 31 \\
\hline
\end{tabular}

Table 2: The evaluations of hyperintense ON at T2WI by 2 radiologists

\begin{tabular}{lccc}
\hline & \multicolumn{3}{c}{ Radiologist A } \\
\cline { 2 - 4 } Radiologist B & Absent & Present & Total \\
\hline Absent & 35 & 5 & 40 \\
Present & 0 & 22 & 22 \\
Total & 35 & 27 & 62 \\
\hline
\end{tabular}

$0.21-0.40$, moderate for $\kappa$ values of $0.41-0.60$, good for $\kappa$ values of $0.61-0.80$, and excellent for $\kappa$ values of $\geq 0.81{ }^{18}$ The percentage agreement between measurements (tumor height and ONKA) from the radiologists was also calculated by concordance correlation coefficients and $95 \%$ confidence intervals. ${ }^{19}$ To determine the variables associated with the visual impairment before the operation and postoperative improvement, we used a univariate binary logistic regression analysis using generalized estimating equations that adjusted for laterality of the optic pathway. The variables included the clinical (age, sex, and duration of symptoms) and imaging (tumor height, chiasmal compression, hyperintense ONs on T2WI, and ONKA) characteristics. $P$ values $<.05$ indicated statistical significance. For the ONKA, we calculated the sensitivity, specificity, and accuracy for the prediction of postoperative improvement. For this analysis, we used cutoff values in a receiver operating characteristic curve analysis, which were determined with the Youden index. ${ }^{20}$

\section{RESULTS}

In all cases, 1 radiologists could detect the optic pathway with CE-FIESTA. Radiologists A and B scored 20 (65\%) and 22 (71\%) of 31 optic chiasms as severe chiasmal compression, respectively (Table 1). On the basis of the ratings of radiologists A and B, hyperintense ONs on T2WI were seen in 27 (44\%) of 62 sides and $22(35 \%)$ sides, respectively (Table 2$)$. On CE-FIESTA, the radiologists found an area of abnormal high signal intensity in the center of the right optic tract in only 1 patient, who had little improvement of visual impairment after the operation. Therefore, we excluded the finding of hyperintense ONs on CE-FIESTA from all analyses because it was thought to be of infrequent incidence.

The presence of chiasmal compression and hyperintense ONs on T2WI was shown to have moderate and excellent interobserver agreement, with $\kappa$ values of 0.56 and 0.83 , respectively. Interobserver reproducibility of measurements by the 2 radiologists demonstrated 99\% agreement for tumor height (95\% CI, 97\%-99\%) and $98 \%$ for ONKA (95\% CI, 69\%-99\%).

The multivariate logistic regression analyses showed that hyperintense ON $(P=.020)$ and ONKA $(P=.030)$ were independently associated with the presence of VAD before the operation (On-line Table 1). The multivariate logistic regression analyses showed that the chiasmal compression severity $(P=.047)$ was 
independently associated with the presence of VFD before the operation (On-line Table 1).

The postoperative ophthalmologic examinations were obtained, on average, 14.8 days (range, 4-92 days) after the operathe operation, we found good improvement after the operation in 17 (59\%) and $32(63 \%)$ sides, respectively. For VAD, the univariate and multivariate analyses revealed that only ONKA $(P=.009$ and $P=$ .011 , respectively) was significantly associated with postoperative improvement (On-line Table 2 and Figs 2 and 3). For VFD, the multivariate analysis revealed that only ONKA $(P=.002)$ was significantly associated with postoperative improvement (On-line Table 2). tion. Of the 29 sides with VAD and the 51 sides with VFD before

The receiver operating characteristic analysis suggested optimal cutoff levels for the ONKA ( $\geq 102.5$ for VAD and $\geq 114.5^{\circ}$ for VFD) for prediction of the postoperative improvement. Therefore, the sensitivity, specificity, and accuracy for the prediction of postoperative improvement with ONKA were 88\% (15/17), 92\% (11/12), and 90\% (26/29) for VAD and 72\% (23/32), 89\% (17/ $19)$, and $78 \%$ (40/51) for VFD, respectively (Table 3 ).

\section{DISCUSSION}

Multivariate and univariate analyses demonstrated that the degree of ONKA was the only independent predictor of postoperative improvement for VAD and VFDs. Other MR imaging findings were
associated with visual impairment before
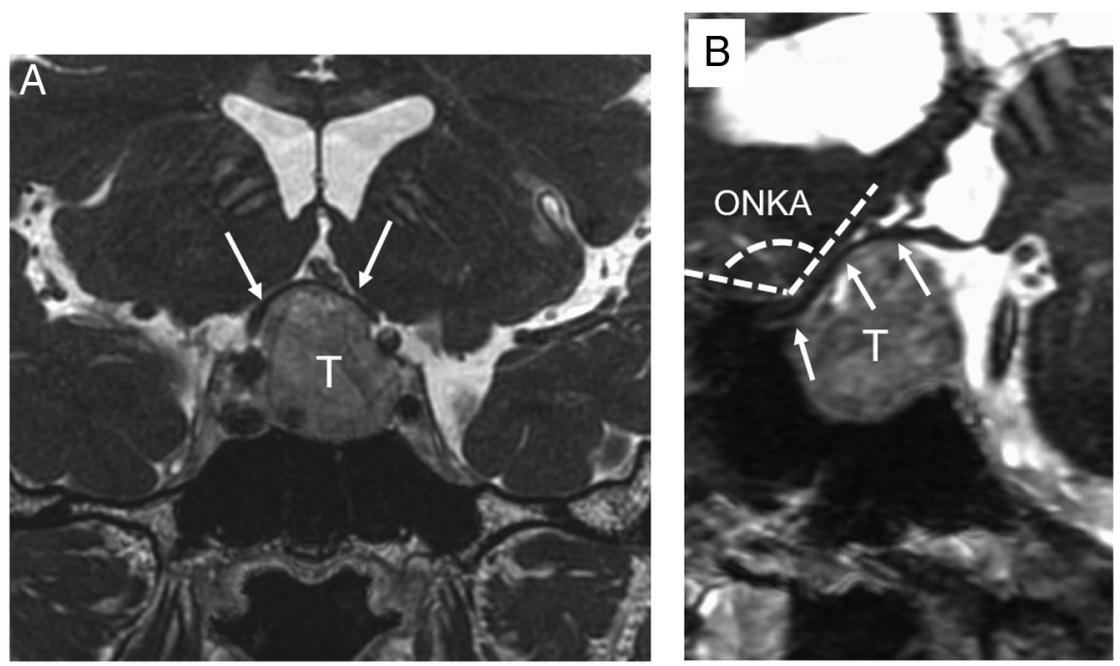

FIG 2. Images from a 53-year-old man with a pituitary macroadenoma who presented with left visual acuity disturbance (20/50 vision on the Snellen Eye Chart) before the operation. After the operation, the left visual acuity disturbance was improved (20/20 vision on the Snellen Eye Chart). $A$, A coronal CE-FIESTA image shows severe chiasmal compression (arrows) by the pituitary macroadenoma. $B$, A sagittal oblique CE-FIESTA image demonstrates a left optic nerve kinking angle of $120^{\circ}$. The arrows indicate the left optic nerve. T indicates pituitary macroadenoma. the operation but not with its postoperative improvement.

The ONKA with an optimal cutoff level had a high specificity for the prediction of postoperative improvement of VAD and VFD. This result indicates that patients with ONKA of $<114.5^{\circ}$ may have potential irreversible damage to the ON. Regarding this irreversible damage to the ON at the optic canal orifice, we speculate 2 possible mechanisms: 1) direct damage due to ON compression from the falciform ligaments, and 2) hemodynamic complications affecting the $\mathrm{ON}$ induced by the kink. In large suprasellar tumors, the ONs have been compressed by the falciform ligament at the optic canal orifice. ${ }^{21}$ Therefore, the decompression procedure of the optic canal consists of extradural anterior clinoidectomy with falciform lig-
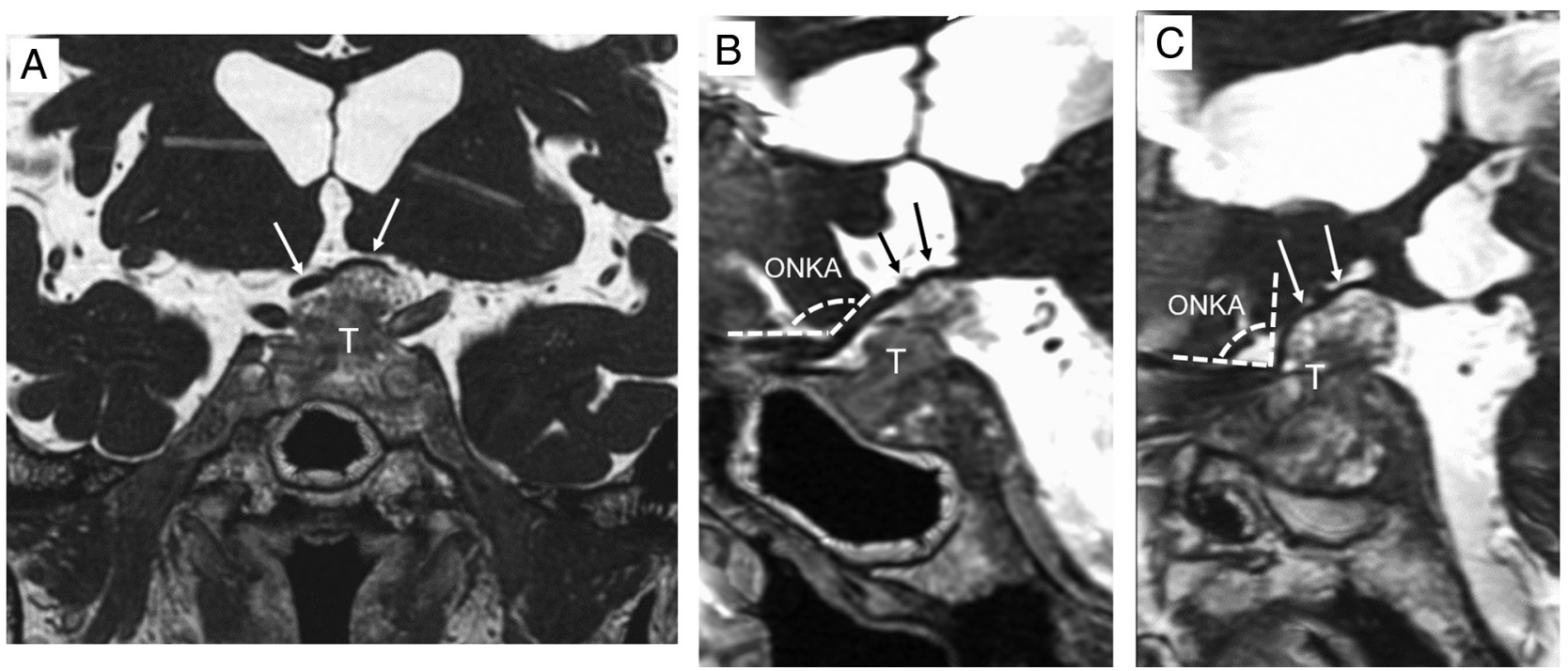

FIG 3. Images from a 75-year-old man with a pituitary macroadenoma who presented with a bilateral visual acuity disturbance (right: $20 / 50$ and left: $20 / 70$ vision on the Snellen Eye Chart, respectively) before the operation. After the operation, the left visual acuity disturbance was not improved (20/50 vision), though the right visual acuity disturbance was improved (20/30 vision). Left optic atrophy (disc pallor) was noted on a postoperative assessment by an ophthalmologist, but there were negative findings on the preoperative assessment. $A$, A coronal CE-FIESTA image shows moderate chiasmal compression by the pituitary macroadenoma. A sagittal oblique CE-FIESTA image demonstrates a right optic nerve (arrows) kinking angle of $133^{\circ}(B)$ and a left optic nerve (arrows) kinking angle of $90^{\circ}(C)$. T indicates pituitary macroadenoma. 
Table 3: ONKA with CE-FIESTA — correlation with indicators of postoperative good improvement ${ }^{\mathrm{a}}$

\begin{tabular}{lcc} 
& \multicolumn{1}{c}{ VAD } & VFD \\
\hline Cutoff values for the ONKA & $\geq 102.5^{\circ}$ & $\geq 114.5^{\circ}$ \\
Sensitivity & $88(15 / 17)$ & $72(23 / 32)$ \\
Specificity & $92(11 / 12)$ & $89(17 / 19)$ \\
Accuracy & $90(26 / 29)$ & $78(40 / 51)$ \\
\hline
\end{tabular}

${ }^{a}$ Data are percentages. Numbers in parentheses were used to calculate the percentages.

ament and optic nerve sheath opening. ${ }^{21}$ Regarding the hemodynamic complications, Hoyt ${ }^{22}$ reported that $\mathrm{ON}$ compression due to pituitary macroadenoma disrupts the arterial supply, and longterm compression of the arteries, veins, and capillary networks in the ON leads to stagnant anoxia. We believe that these mechanisms have resulted in a cumulative or additive effect for irreversible damage to the $\mathrm{ON}$.

The ONKA measurement as a quantitative evaluation demonstrated excellent interobserver agreement, possibly due to the high accuracy of CE-FIESTA in depicting the anterior optic pathways. For ON depiction in patients with pituitary macroadenoma, CE-FIESTA has several advantages over conventional MR images - namely the high spatial resolution and defined contrast difference between the tumor and surrounding structures, such as the ON, CSF, and skull bone. ${ }^{5}$ The spatial resolution with CEFIESTA is $0.6 \times 0.6 \times 0.8 \mathrm{~mm}$, and 3D CE-FIESTA data also allow radiologists to examine the entire optic pathway contiguously. Furthermore, CE FIESTA images provide good contrast of the optic pathway relative to enhanced tumor and CSF, because the optic pathway has a low signal intensity, whereas the enhanced tumor and CSF have high signal intensity. Conversely, the contrast difference between the ON and tumors with T2WI may decrease due to hyperintense ONs caused by compression by the tumor. For CE-T1WI, the poor visibility of the optic pathways may be due to poor contrast between the CSF and the optic pathways; both may be hypointense on CE-T1WI. Therefore, our assessment with CE-FIESTA has clinical importance in that it may provide a simple and reproducible method for evaluating the irreversible visual impairment in patients with a pituitary macroadenoma.

Regarding the association between the duration of symptoms and the postoperative improvement of VFD, the results from previous studies are inconsistent, ${ }^{2-4,23}$ and we found no association between them. One possible explanation for our result may be due to poor subjective symptoms caused by pituitary macroadenomas because of their slow growth. Hyperintensity of the ONs was also not associated with irreversible damage to the $\mathrm{ON}$. Our results are supported by the findings of a previous report ${ }^{5}$; for the prediction of irreversible damage to the ONs after an operation in a large suprasellar tumor, hyperintense ONs on T2WI had good sensitivity (100\%) but poor specificity (42\%), suggesting the possibility of not only degeneration of the ONs but also reversible damage as an etiologic factor in the hyperintense ONs.

Although VFDs are well-known to be caused by chiasmal compression, we found that the degree of chiasmal compression was not associated with irreversible damage to the ONs. Our results indicate that for reflecting damage to the ONs, an ON kink at the optic canal orifice may be a more important factor than chiasmal compression. In a previous study, hyperintensity of the optic pathways on CE-FIESTA had high accuracy in predicting irreversible damage to the ONs. ${ }^{5}$ Consistent with the findings from this previous report, we found 1 patient with hyperintensity on CEFIESTA who had little improvement of visual impairment after the operation. However, this finding seems to be of infrequent incidence in patients with pituitary macroadenomas. The previous study also demonstrated that an optic nerve size on MR imaging is an important predictive factor for vision recovery. ${ }^{15}$ However, we excluded this finding from all analyses because there was no case with obvious optic nerve atrophy on MR imaging. The main reason seems to be that almost all our cases included patients with a relatively shorter duration of vision loss. Our data indicate that optic nerve atrophy on MR imaging may be a finding in advanced phases of compressive neuropathy.

Our study has several limitations. First, the postoperative ophthalmologic examinations were performed at various intervals after an operation. Moreover, in the current study, the mean time to postoperative ophthalmologic examinations was relatively short (14.9 days). One previous report showed that postoperative improvement of the VFD was progressive and apparent even at the 5-year follow-up. ${ }^{4}$ Therefore, this finding may lead to overestimation of the association between the ONKA and the postoperative improvement of visual impairment. Further prospective longterm follow-up studies, including thorough clinical investigations, are needed to determine the clinical impact of the finding of the ONKA. Second, we evaluated VFDs using the ophthalmologic examination, in which, however, false-positives and false-negatives can occur with the perimetry, depending on patient cooperation. Chouinard et $\mathrm{al}^{24}$ demonstrated that longitudinal changes in visual field perimetry before and after the operation correlated with those in fMRI activation in a retinotopic manner. Because a retinotopic examination with $\mathrm{fMRI}$ is passive and more objective, in a further study, the use of fMRI may allow a more accurate evaluation of VFD.

\section{CONCLUSIONS}

In our logistic analyses, the only independent predictor of postoperative improvement of visual impairment in patients with pituitary macroadenomas was the degree of ONKA. Our results indicate that early irreversible damage to the ONs is associated with kinking at the optic canal orifice caused by compression of the pituitary macroadenomas. Our quantitative assessment with CE-FIESTA with high excellent interobserver agreement may provide useful information for managing patients with pituitary macroadenomas. This study suggests that CE-FIESTA is indeed a beneficial and practical MR imaging method for the preoperative evaluation of pituitary macroadenomas.

\section{REFERENCES}

1. Fahlbusch R, Honegger J, Buchfelder M. Surgical management of acromegaly. Endocrinol Metabol Clin North Am 1992;21:669-92 Medline

2. Powell M. Recovery of vision following transsphenoidal surgery for pituitary adenomas. Br J Neurosurg1995;9:367-74 Medline

3. Peter M, De Tribolet N. Visual outcome after transsphenoidal surgery for pituitary adenomas. Br J Neurosurg 1995;9:151-57 CrossRef Medline

4. Gnanalingham K, Bhattacharjee S, Pennington R, et al. The time

AJNR Am J Neuroradiol 38:2067-72 Nov 2017 www.ajnr.org

2071 
course of visual field recovery following transphenoidal surgery for pituitary adenomas: predictive factors for a good outcome. J Neurol Neurosurg Psychiatry 2005;76:415-19 CrossRef Medline

5. Watanabe K, Kakeda S, Yamamoto J, et al. Delineation of optic nerves and chiasm in close proximity to large suprasellar tumors with contrast-enhanced FIESTA MR imaging. Radiology 2012;264: 852-58 CrossRef Medline

6. Lee IH, Miller NR, Zan E, et al. Visual defects in patients with pituitary adenomas: the myth of bitemporal hemianopsia. AJR Am J Roentgenol 2015;205:W512-18 CrossRef Medline

7. Margalit NS, Lesser JB, Moche J, et al. Meningiomas involving the optic nerve: technical aspects and outcomes for a series of 50 patients. Neurosurgery 2003;53:523-33; discussion 532-33 CrossRef Medline

8. Liu JK, Christiano LD, Patel SK, et al. Surgical nuances for removal of tuberculum sellae meningiomas with optic canal involvement using the endoscopic endonasal extended transsphenoidal transplanum transtuberculum approach. Neurosurg Focus 2011;30:E2 CrossRef Medline

9. Haacke EM, Wielopolski PA, Tkach JA, et al. Steady-state free precession imaging in the presence of motion: application for improved visualization of the cerebrospinal fluid. Radiology 1990;175: 545-52 CrossRef Medline

10. Tsuchiya K, Aoki C, Hachiya J. Evaluation of MR cisternography of the cerebellopontine angle using a balanced fast-field-echo sequence: preliminary findings. Eur Radiol 2004;14:239-42 CrossRef Medline

11. Chung HW, Chen CY, Zimmerman RA, et al. T2-weighted fast MR imaging with true FISP versus HASTE: comparative efficacy in the evaluation of normal fetal brain maturation. AJR Am J Roentgenol 2000;175:1375-80 CrossRef Medline

12. Jayakumar PN, Kovoor JM, Srikanth SG, et al. 3D steady-state MR cisternography in CSF rhinorrhoea. Acta Radiol 2001;42:582-84 CrossRef Medline

13. Shigematsu Y, Korogi Y, Hirai T, et al. Contrast-enhanced CISS MRI of vestibular schwannomas: phantom and clinical studies. J Comput Assist Tomogr 1999;23:224-31 CrossRef Medline
14. Davagnanam I, Chavda SV. Identification of the normal jugular foramen and lower cranial nerve anatomy: contrast-enhanced 3D fast imaging employing steady-state acquisition MR imaging. AJNR Am J Neuroradiol 2008;29:574-76 CrossRef Medline

15. Carlson AP, Stippler M, Myers O. Predictive factors for vision recovery after optic nerve decompression for chronic compressive neuropathy: systematic review and meta-analysis. J Neurol Surg $B$ Skull Base 2013;74:20-38 CrossRef Medline

16. McGraw P, Winn B, Whitaker D. Reliability of the Snellen chart. BMJ 1995;310:1481-82 CrossRef Medline

17. Levin LA. Topical diagnosis of chiasmal and retrochiasmal disorders. In: Miller NR, Walsh FB, Hoyt WF, eds. Walsh and Hoyt Clinical Neuro-Ophthalmology. 6th ed. Baltimore: Lippincott Williams \& Wilkins; 2005:503-73

18. Landis JR, Koch GG. The measurement of observer agreement for categorical data. Biometrics 1977;33:159-74 CrossRef Medline

19. Bernard SA, Murphey MD, Flemming DJ, et al. Improved differentiation of benign osteochondromas from secondary chondrosarcomas with standardized measurement of cartilage cap at CT and MR imaging. Radiology 2010;255:857-65 CrossRef Medline

20. Youden WJ. Index for rating diagnostic tests. Cancer 1950;3:32-35 CrossRef Medline

21. Sade B, Lee JH. High incidence of optic canal involvement in tuberculum sellae meningiomas: rationale for aggressive skull base approach. Surg Neurol 2009;72:118-23; discussion 123 CrossRef Medline

22. Hoyt W. Correlative functional anatomy of the optic chiasm. 1969. Clin Neurosurg 1970;17:189-208 Medline

23. Cohen AR, Cooper PR, Kupersmith MJ, et al. Visual recovery after transsphenoidal removal of pituitary adenomas. Neurosurgery 1985; 17 ; 446-52 CrossRef Medline

24. Chouinard PA, Striemer CL, Ryu WH, et al. Retinotopic organization of the visual cortex before and after decompression of the optic chiasm in a patient with pituitary macroadenoma: case report. J Neurosurg 2012;117:218-24 CrossRef Medline 